\title{
Cursive and gelastic epilepsy: probable sequelae of physical abuse
}

\author{
T. H. WooN* \\ M.D. \\ V. VIGNAENDRA \\ M.B., B.S., F.R.A.C.P. \\ Departments of Psychological Medicine and Medicine, University Hospital, Kuala Lumpur, Malaysia
}

\begin{abstract}
Summary
An abandoned 8-year-old male child, who was subjected to physical abuse, presented with abnormal behaviour and focal seizures. The focal seizures included involuntary running attacks (epilepsia cursiva) and uncontrollable laughter (gelastic epilepsy). The occurrence of cursive and gelastic seizures in the same patient is extremely rare. These fits were probably a late sequel of head trauma.
\end{abstract}

\section{Introduction}

Episodic attacks of running and uncontrollable laughter, in persons with disturbed consciousness, are recognized as rare epileptic manifestations and are called cursive seizures (Sisler, Levy and Roseman, 1953) and gelastic epilepsy (Daly and Mulder, 1957) respectively. Chen and Forster (1973) found that only $0.32 \%$ of their seizure population had either gelastic or cursive fits. It was extremely rare for both seizures to occur in the same patient. In battered children sustaining head injuries, $1-5 \%$ develop post-traumatic seizures (Till, 1975).

A child is now described who was battered, and manifested both gelastic and cursive seizures which were a probable late sequel of head trauma.

\section{Case report}

In May 1970, an 8-year-old boy was seen at the Psychiatry service of the University Hospital, Kuala Lumpur, for abnormal behaviour. The child had pica, was hyperactive, anti-social, aggressive and was described as attention-seeking. In addition, he suffered weekly focal fits. These consisted of the right arm becoming stiff and flexed at the elbow with eyes upturned and lips pulled to the right. Each fit lasted about $30 \mathrm{sec}$ and he had amnesia for it. He often fell asleep after the fit. By 1971, his focal seizures included daily attacks consisting of an aura of abnormal sensation in the throat followed by loss of consciousness and accompanied by clonic jerks of the upper limbs and post-ictal confusion.

In 1974, at the age of 12 years, new patterns of fits

\footnotetext{
* Correspondence to: T. H. Woon, M.D. Department of Psychological Medicine, Faculty of Medicine, University of Malaya, Kuala Lumpur, 22-11, Malaysia.
}

were noted. With his right arm held flexed, he would suddenly run around for several seconds. There was no incontinence or tongue-biting but he appeared to be unaware of these seizures. Ten such episodes were noted over a 2-month period. About this time he had a third type of seizure which consisted of sudden bursts of uncontrollable laughter lasting a few seconds. He was observed to press his cheeks with his right hand as if trying to control his laughter. He would sit in one place during these seizures. When questioned later, he was unable to recall the attacks. They were noticed to occur once or twice each day.

His alcoholic father had deserted the family. The mother, who was supposed to have ill-treated him and the other siblings, abandoned him to a cowherd. This cowherd is said to have physically abused the patient. Just before his referral to the University Hospital, he was severely abused by being hung by the ankles over a pile of smoking firewood.

Examination showed septic scalp wounds infested with maggots. These healed to leave old scars which were 2 inches long over the occipital region, 1 inch long over the left parietal region and 2 inches long over the left frontal region. Apart from being slightly emaciated, he had no other physical abnormality. A psychological assessment showed a verbal IQ of 55 , a performance IQ of 53 and a full-scale IQ of 50. Qualitatively, his performance resembled that of a child who had experienced social and environmental deprivation.

Initial control of his fits was attempted with primidone and carbamazepine. His behaviour improved slightly with personal care in a small foster home with eight children. In 1974, his medication was gradually changed to phenytoin $100 \mathrm{mg}$ twice/ day and this brought the seizures under control.

His serum sodium, potassium, chloride, magnesium, calcium, phosphate, urea and fasting blood sugar were within normal limits. His blood serology for syphilis was unreactive.

\section{Electroencephalographic findings}

EEGs were done with a 16-channel Beckman machine using nineteen scalp electrodes placed 
according to the 10-20 system. Recordings were done at a paper speed of $30 \mathrm{~mm} / \mathrm{sec}$ and a time constant of $0.3 \mathrm{sec}$.

The initial tracing in 1970 showed a 9 cycles per second (c.p.s.) alpha rhythm and arrhythmic 3-6 c.p.s. waves over the temporal areas of the left hemisphere. Numerous sharp wave or spike and wave epileptic discharges were seen over the left temporal regions. In addition, epileptic discharges appeared over the fronto-temporal regions, usually bisynchronously but with left-sided preponderance.

An EEG in 1971 (Fig. 1) similarly showed slow waves and numerous epileptic discharges over the left temporal regions. In a repeat tracing in late 1974, the slow wave abnormalities over the left temporal areas were less prominent, sharp waves and multiple spike and wave epileptic discharges occurred over the left fronto-temporal regions which sometimes simultaneously involved the corresponding areas of the right hemisphere (Fig. 2). On this occasion, the beta rhythms were reduced over the left anterior head regions.

\section{Discussion}

The multiple scalp scars in this child are evidence that he had suffered head injuries. Courville (1958) demonstrated that in closed head injuries, contrecoup brain contusions tend to be localized to the basal surface of the frontal lobes and the basal, dorso-lateral and polar regions of the temporal lobes since the irregular surface over the anterior and middle cranial fossae preferentially contuse these areas of the brain as it moves over them. The focal EEG abnormalities in the present patient suggest that left fronto-temporal areas had probably sustained the contusions and had later matured into an epileptic focus.

The episodes of uncontrollable laughter and running attacks in this child are seizures since each attack was stereotyped, its onset abrupt and without

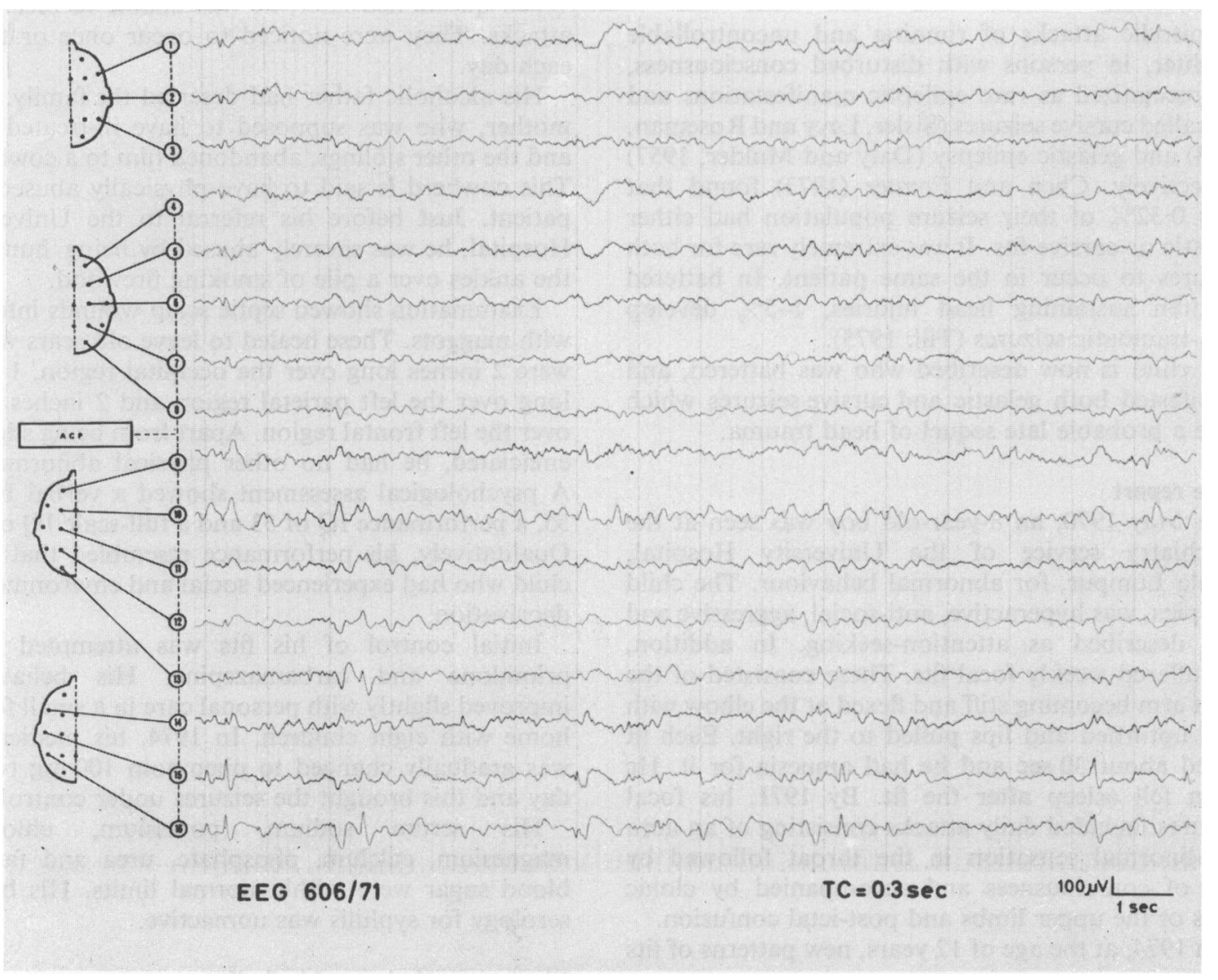

Fig. 1. Epileptic discharges in the left middle and posterior temporal regions. Prominent arrhythmic delta waves over the same areas (1971). 

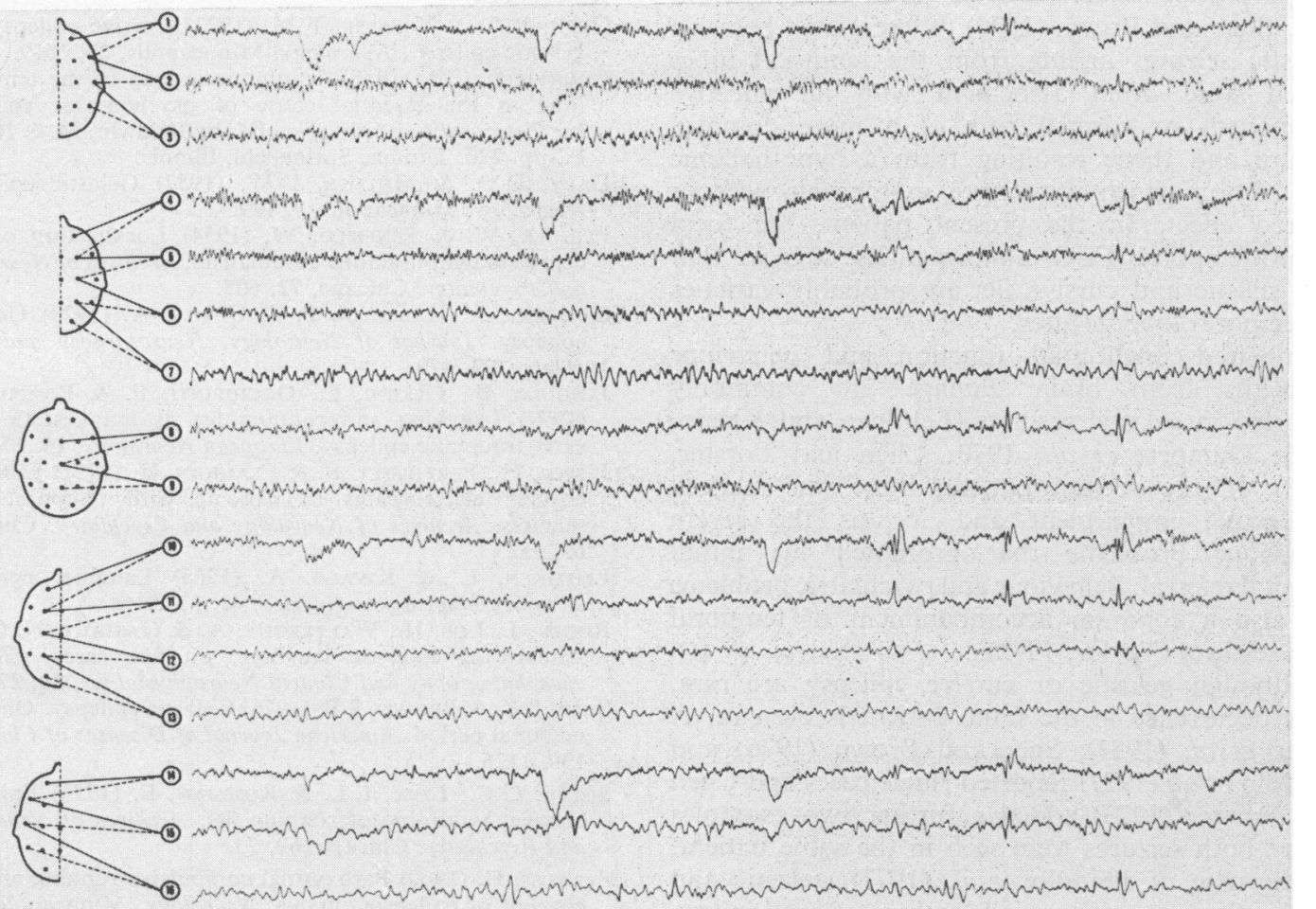

EEG $756 / 74$

$T C=0.3 \mathrm{sec}$

$50 \mu \mathrm{V} \underbrace{}_{1 \mathrm{sec}}$

FIG. 2. Slow wave abnormalities in the left temporal regions are improved. Numerous epileptic discharges in the left fronto-temporal areas. Beta rhythms are attenuated over the left anterior head regions (1974).

known precipitating factors and the patient had no awareness for the events. The interictal epileptic discharges in the EEG and the control of the episodes of running attacks and uncontrollable laughter with phenytoin further support the epileptic nature of these repetitive attacks. Not all patients with gelastic or cursive seizures, however, remain unconscious during the ictus; nonetheless, these patients later recall that they were unable to control the running, or 'forced' laughter, or failed to experience the amusement that usually accompanies laughter (Strauss, 1960; Lehtinen and Kivalo, 1965).

The site of the brain pathology in gelastic epilepsy is controversial. In reviewing the literature on gelastic epilepsy and pathological laughter, Gumpert, Hansotia and Upton (1970) found evidence to implicate abnormalities in the hypothalamic region. Sher and Brown (1976), indeed, reported two cases of gelastic seizures, one of whom also had epilpesia cursiva, associated with diencephalic or interpeduncular mass lesions. The great variability in the interictal EEG abnormalities in gelastic seizures, (Gumpert et al., 1970; Chen and Forster, 1973) however, does not readily allow for a consistent cortical localization of these fits. Nevertheless, the frequent association of gelastic epilepsy with psychomotor seizures and with temporal lobe focal EEG abnormalities in their series, led Chen and Forster (1973) to conclude that lesions with widespread origin in the brain might influence a common focus in the temporal lobe or the limbic system to induce gelastic epilepsy. EEG recordings during gelastic seizures in three patients (Roger et al., 1967) showed temporal lobe focal origin in all, implicating the temporal lobe in these fits. In the case reported by Gumpert et al. (1970), the EEG during gelastic status demonstrated electro-decremental activity which is known to be the electrical correlate of deep seated temporal lobe electrical seizures (Jasper, Pertuisset and Flanigin, 1951; Feindel and Penfield, 1954). However, it is also possible that in an individual patient, either the hypothalamus or the 
temporal lobe is the site of origin of gelastic fits; in fact, Sher and Brown (1976) differentiated between gelastic seizures arising from the temporal lobes which were often associated with an affective component or formed part of a more complex seizure and those resulting from a hypothalamic dysfunction which were pure motor acts without altered effect. In the present patient, the focal fronto-temporal EEG abnormalities suggest that the gelastic and cursive fits are probably varieties of temporal lobe seizures.

Impaired intellectual function and behaviour problems from brain damage are commonly reported in gelastic seizures (Lehtinen and Kivalo, 1965; Gumpert et al., 1970; Chen and Forster, 1973). In the present patient, there was marked intellectual impairment and it was the severe behaviour problems that prompted his initial hospital referral. Behaviour and psychiatric problems are also a common accompaniment of temporal lobe seizures (Taylor, 1972).

Although gelastic or cursive epilepsy are rare, their occurrence in the same patient is even rarer. Sisler et al. (1953), Sher and Brown (1976) and Jandolo et al. (1977) reported single cases and Chen and Forster (1973) added a further two examples where both seizures were seen in the same patient. In the case of Jandolo et al. (1977), gelastic and cursive fits were early manifestations of head injury. The present case is another rare example where both types of fit occurred in the same patient and were probably a late sequel of head injury.

\section{Acknowledgments}

We thank the Medical Illustration Unit, Faculty of Medicine, University of Malaya, for preparing the 2 figures.

\section{References}

Chen, R.C. \& Forster, F.M. (1973) Cursive epilepsy andQ gelastic epilepsy. Neurology. Minneapolis, 23, 1019.

Courville, C.B. (1958) Traumatic lesions of the temporallobe as the essential cause of psychomotor epilepsy. $\overline{5}$ In: Temporal Lobe Epilepsy, Ed. by Baldwin, M. \& Bailey P., pp. 220. Thomas Springfield, Illinois.

Daly, D.D. \& Mulder, D.W. (1957) Gelastic epilepsy $\frac{\bar{\sigma}}{\bar{\sigma}}$ Neurology. Minneapolis, 7, 189.

Feindel, W. \& Penfield, W. (1954) Localization of dis-疋 charge in temporal lobe automatism. Archives of Neurology
and Psychiatry. Chicago, 72, 605.

Gumpert, J., Hansotia, P. \& Upton, A. (1970) Gelastic epilepsy. Journal of Neurology, Neurosurgery and Psychiatry, 33, 479.

Jandolo, B., Gessini, L., Occhipinti, E. \& Pompili, A. (1977) Laughing and running fits as manifestations of early traumatic epilepsy. European Neurology, 15, 177.

Jasper, H., Pertuisset, B. \& Flanigin, H. (1951) EEG and3. cortical electrograms in patients with temporal lobecr seizures. Archives of Neurology and Psychiatry. Chicago, 65, 252.

Lehtinen, L. \& Kivalo, A. (1965) Laughter epilepsy. Acta neurologica scandinavica, 41, 225.

Roger, J., LoB, H., Waltregny, A. \& Gastaut, N. (1967) Attacks of epileptic laughter; on five cases. Electro-음 encephalography and Clinical Neurophysiology, 22, 279.

SHER, P.K. \& Brown, S.B. (1976) Gelastic epilepsy. Onset in neonatal period. American Journal of Diseases of Children, 130, 1126.

Sisler, G.C., Levy, L.L. \& Roseman, E. (1953) Epilepsia $\frac{\Phi}{3}$ cursiva, syndrome of running fits. Archives of Neurolo $y$. and Psychiatry. Chicago, 69, 73.

Strauss, H. (1960) Paroxysmal compulsive running and 蛅e $\overrightarrow{0}$ concept of epilepsia cursiva. Neurology. Minneapolis, 341.

TAYLOR, D.C. (1972) Mental state and temporal lobe epilepsy. A correlative account of 100 patients treated surgically. Epilepsia, 13, 727.

TILl, K. (1975) A neurosurgeon's viewpoint. In: Concerning Child Abuse. Papers presented by the Tunbridge Wells $\propto$ Study Group on non-accidental injury to children, Ed. by Franklin, A.W., p. 60. Churchill-Livingstone, London. $\overrightarrow{\bar{B}}$ 